Pure and Applied Mathematics Quarterly

Volume 3, Number 4

(Special Issue: In honor of

Grisha Margulis, Part 1 of 2)

987-1003, 2007

\title{
Free Subgroups of Linear Groups
}

\author{
G.A. Soifer
}

To Gregory Margulis in his birthday

In the celebrated paper [T] J. Tits proved the following fundamental dichotomy for a finitely generated linear group :

Let $G$ be a finitely generated linear group over an arbitrary field. Then either $G$ is virtually solvable or $G$ contains a free non-abelian subgroup.

His proof of this alternative based on geometrical ideas came form Schottky groups. Recall that a Schottky group $G$ is a group of fractional linear transformations of the hyperbolic plane $\mathbb{H}^{2}$ generated by a set of hyperbolic elements $S=$ $\left\{g_{i}, i \in I\right\}$ and has the following property : there exist disjoint subsets $D_{i}^{ \pm}, i \in I$ and $D^{0}$ of $\mathbb{H}^{2}$ such that for every $i \in I$ :

1. $g_{i}^{n}\left(\cup_{j \in I, j \neq i} D_{j}^{ \pm} \cup D^{0}\right) \subseteq D_{i}^{+}$for $n>0$

2. $g_{i}^{n}\left(\cup_{j \in I, j \neq i} D_{j}^{ \pm} \cup D^{0}\right) \subseteq D_{i}^{-}$for $n<0$.

Note that from the definition immediately follows that the group $G$ is a free group with free generators $g_{i}, i \in I$. Indeed, let $g=g_{i_{1}}^{m_{1}} \ldots g_{i_{k}}^{m_{k}}$ be any reduced word. Take $p \in D^{0}$, then $g p \in D_{i_{1}}^{ \pm} \subseteq \mathbb{H}^{2} \backslash D^{0}$. Therefore $g \neq 1$.

One of the main purposes of the present work is to show how the beautiful ideas of Tits were developed in our joint works with G. Margulis [MS1], [MS2], [MS3]. Our interest to free subgroups of linear groups was initiated by the following Problem 1 ( $V$. Platonov) Does there exist a maximal subgroup of infinite index in $S L_{n}(\mathbb{Z})$ for $n \geq 3$ ?

We proved in [MS1] that the answer is positive. Actually we proved that

Received April 11, 2006. 
Let $G$ be a finitely generated linear group over an arbitrary field. Then either $G$ is polycyclic or $G$ contains a maximal subgroup of infinite index.

We proved this as a corollary of the following theorem:

Let $G$ be a finitely generated linear group over an arbitrary field. If $G$ is not virtually solvable then $G$ contains a pro-finitely dense free subgroup.

We remark that questions about existence of a dense free subgroup in topological groups are very important and leads to a many deep consequences (see for example [BG2]).

Conjecture ( $[D P S S],[P])$. Let $G$ be a finitely generated linear group and let $\widehat{G}$ be a pro-finite completion of the group $G$. Assume that $G$ contains a free subgroup. Then does $\widehat{G}$ contain a finitely generated pro-finitely dense free subgroup?

E. Breuillard and T. Gelander proved this conjecture in [BG2]

A. Shalev asked me a slightly different question. Namely

Problem 2 (A. Shalev). Let $G$ be a finitely generated linear group and let $\widehat{G}$ be the pro-finite completion of the group $G$. Assume that $G$ contains a free subgroup. Does $G$ contain a finitely generated free subgroup which is pro-finitely dense in $\widehat{G}$ ?

In general an answer to this question is negative. For example it is not true if $G$ is a lattice in a semisimple Lie group of real rank 1. But for lattices in higher rank semisimple groups the answer is positive $[\mathrm{SV}]$.

Several resent very interesting results [BG1],[BG2],[GG1] were proved under the influence of [MS3]. Therefore we decided to use this opportunity to explain some modifications of the concept, ideas and proofs from this paper together with further development done in our works [AMS1], [AMS2]. Based on this, we will give a short proof of Platonov's problem, construct a new counterexample to a Prasad-Tits conjecture and state some new and recall some old problems.

In the last section we will complete a proof of the following:

Conjecture (G. Margulis) Let $S$ be a crystallographic semigroup, then $S$ is a group.

Our proof of the above conjecture is based on existence of a free subgroup in the Zariski closure of $S$ with some additional geometric properties. This step was done in $[\mathrm{S}]$.

We will use standard definitions of algebraic group theory, Lie group theory and group theory (see $[\mathrm{B}],[\mathrm{H}],[\mathrm{R}]$ ). The letters $\mathbb{Z}, \mathbb{Q}, \mathbb{R}, \mathbb{C}, \mathbb{Q}_{p}$ denotes respectively the set of integers, rational, real, complex and $p$-adic numbers. The index of a subgroup $H$ of a group $G$ will be denoted by $|G / H|$. If $G$ is a group and $S \subseteq G$, then by $N_{S}(G)$ ( resp. $C_{S}(G)$ ) we denote the normalizer (resp. centralizer) of $S$ in $G$. By $\langle S\rangle$ we denote the subgroup of $G$ generated by $S$. We denote as usual 
by $G L_{n}$ and $S L_{n}$ the group of invertible and unimodular $n$ by $n$ matrices. An algebraic subgroup of $G L_{n}$ defined over a field $k$ is called an algebraic $k$-group or a $k$-group. The set of all $k$-points of an algebraic variety $W$ will be denoted by $W(k)$. If $\varphi: G \longrightarrow H$ is a $k$-rational homomorphism of $k$-groups $G$ and $H$ and the field $l$ is an extension of $k$ then the natural homomorphism $\varphi(l): G(l) \longrightarrow$ $H(l)$ will be denote by $\varphi$ as well. Let $V$ be a finite dimensional vector space. A subgroup $G$ of $G L(V)$ is called irreducible if there is no proper $G$ invariant subspace of $V$. Accordingly, we call a representation $\rho: G \longrightarrow G L(V)$ irreducible if the image $\rho(G)$ is a irreducible subgroup of $G L(V)$. A representation $\rho: G \longrightarrow$ $G L(V)$ is called strongly irreducible if for every subgroup $H$ of finite index of $G$ the group $\rho(H)$ is irreducible. If $k$ is a local field and $W$ is an algebraic $k$-variety then $W(k)$ has two natural topologies, namely induced by the topology of $k$ and by the Zariski topology of $W$. In case it will be necessary to avoid confusion the second topology will be distinguished by the prefix " $k$ " i.e., $k$-open, $k$-dense etc.

G. Margulis became my official adviser in the mid 70's At that time in the former USSR such a step was really non-trivial and might have had many repercussions. I want to express here my deep gratitude to Grisha Margulis for all he gave me as a teacher and as a friend.

Acknowlegment: The author would like to thank several institutions and foundations for their support during the preparation of this paper: SFB 701 in Bielefeld University, ENI in Bar-Ilan University, Yale University, NSF under grant DMS 0244406, USA- Israel Binational Science foundation under BSF grant 2004010,

\section{Ping -Pong and free subgroups.}

We will start from the following proposition which actually comes from Schottky groups and the proof based on the same arguments.

Lemma 1.1. Let $G$ be a group acting on a set $X$. Let $S=\left\{H_{i}, i \in I\right\}$ be a collection of subgroups of $G$ and let $\left\{X_{i}\right\}_{i \in I}$ be a set of disjoint subsets of $X$ and let $x_{0}$ be a point , $x_{0} \in X$. Assume that

1. $x_{0} \in X \backslash \cup_{i \in I} X_{i}$

2. $h_{i}\left(\cup_{j \in I, j \neq i} X_{j}\right) \subseteq X_{i}$ for all $h_{i} \in H_{i}$ and $i \in I$,

3. $h_{i} x_{0} \in X_{i}$ for all $h_{i} \in H_{i}$ and $i \in I$.

Then the group $\langle S\rangle$ is a free product of groups $H_{i}, i \in I$.

Corollary 1.2 (Ping-Pong Lemma) . Let $G, X, S=\left\{H_{i}, i \in I\right\},\left\{X_{i}\right\}_{i \in I}$ and $x_{0}$ 
be as in Lemma 1.1. Assume that for every $i \in I, H_{i}$ is an infinite cyclic group generated by some element $h_{i}$. Then $\langle S\rangle$ is a free group and $h_{i}, i \in I$ are free generators.

It is easy to see that the following statement is true.

Lemma 1.3. Let $G$ be a group and $\rho: G \longrightarrow H$ be a homomorphism of a group.

Let $S=\left\{h_{i}, i \in I\right\}$ be free generators of a free group $\langle S\rangle$. Assume that for every $i \in I$ we choose $g_{i} \in G$ such that $\rho\left(g_{i}\right)=h_{i}$. Then the group generated by $\left\{g_{i}, i \in\right.$ $I\}$ is free and $\left\{g_{i}, i \in I\right\}$ are free generators.

Let $V$ be a finite dimensional vector space over a local field $k$ with absolute value $|\cdot|$ and let $P=\mathbb{P}(V)$ be the projective space based on $V$. Let $g \in G L(V)$ and let $\chi_{g}(\lambda)=\prod_{i=1}^{n}\left(\lambda-\lambda_{i}\right) \in k[\lambda]$ be the characteristic polynomial of the linear transformation $g$. Set $\Omega(g)=\left\{\lambda_{i}:\left|\lambda_{i}\right|=\max _{1 \leq j \leq n}\left|\lambda_{j}\right|\right\}$. Put $\chi_{1}(\lambda)=$ $\prod_{\lambda_{i} \in \Omega(g)}\left(\lambda-\lambda_{i}\right)$ and $\chi_{2}(\lambda)=\prod_{\lambda_{i} \notin \Omega(g)}\left(\lambda-\lambda_{i}\right)$. Since the absolute value of an element is invariant under Galois automorphism then $\chi_{1}$ and $\chi_{2}$ belong to $k[\lambda]$. Therefore $\chi_{1}(g) \in G L(V)$ and $\chi_{2}(g) \in G L(V)$. Let us define by $A(g)$ (resp. $B(g)$ ) the subspace of $P$ corresponding to $\operatorname{ker}\left(\chi_{1}(g)\right)$ (resp. $\left.\operatorname{ker}\left(\chi_{2}(g)\right)\right)$. Put $\operatorname{Cr}(g)=$ $B(g) \cup B\left(g^{-1}\right)$. Recall that $g \in G L(V)$ is called proximal if $A(g)$ is a point. A proximal element $g$ has a unique eigenvalue of maximal absolute value hence this eigenvalue has algebraic and geometric multiplicity one. For $S \subseteq G L(V)$ set $\Omega_{0}(S)=\left\{g \in S: g\right.$ and $\mathrm{g}^{-1}$ are proximal $\}$. We will often use for an element $g \in \Omega_{0}(G L(V))$ the following notation $A(g)=A^{+}(g), B(g)=B^{+}(g), A\left(g^{-1}\right)=$ $A^{-}(g)$ and $B\left(g^{-1}\right)=B^{-}(g)$.

For an element $g \in \Omega_{0}(G L(V))$ the dynamics of the group $\langle g\rangle$ are very transparent. Let us formulate their properties in terms of the projective map $\widehat{g}$ induced by $g$ on the projective space $P$. Namely, the sequence of maps $\widehat{g}^{n}$ where $n$ is positive integer converges to a map sending all points in $P \backslash B(g)$ to the point $A(g)$ and the sequence $\left(\widehat{g}^{-1}\right)^{n}$ where $n$ is positive integer converges to a map sending all points in $P \backslash B\left(g^{-1}\right)$ to the point $A\left(g^{-1}\right)$. This easily follows from the next

Lemma 1.4. Let $g \in \Omega_{0}(G L(V))$ and let $K$ be a compact subset of $P \backslash B(g)$. Let $d$ be the distance between the two compact subsets $K$ and $B(g)$. Assume that $U$ is an open subset in $P$ such that $A(g) \in U$. Then there exits a positive integer $N=N(U, d)$ such that $\widehat{g}^{n} K \subseteq U$ for all $n \geq N$.

To illustrate the dynamics of a subgroup let us conceder the following Example 1. Let $G=S L_{3}(\mathbb{R})$. Let $g_{1}$ and $g_{2}$ be two diagonal matrixes where

$g_{1}=\operatorname{diag}\left(\alpha, \alpha^{-1}, 1\right), \alpha>1$ and $g_{2}=\operatorname{diag}\left(1, \alpha, \alpha^{-1}\right), \alpha>1$. Let $A$ be the abelian group generated by $\left\{g_{1}, g_{2}\right\}$. Clearly $A$ is a free abelian group of a rank 2 . Let $A_{N}=\left\{g_{1}^{n} g_{2}^{m},|n| \geq N,|m| \geq N\right\}$. Put $e_{1}=(1,0,0), e_{2}=(0,1,0), e_{3}=(0,0,1)$. 
Consider the set $\Sigma=\left\{t_{1} e_{1}+t_{2} e_{2}+t_{3} e_{3}, \sum_{1}^{3} t_{i}=1, t_{i} \geq 0, i=1,2,3\right\}$. Let $P(\Sigma)$ be the projectivization of $\Sigma$ and let $\partial P(\Sigma)$ be the boundary of $P(\Sigma)$. Let $q$ (correspondingly, $\left.\widehat{e}_{i} i=1,2,3\right)$ be the point of $P(\Sigma)$ which corresponds to the line span by the vector $1 / 3 e_{1}+1 / 3 e_{2}+1 / 3 e_{3}$ (correspondingly, $\left.e_{i} i=1,2,3\right)$. The boundary $\partial L$ of the orbit $L=\widehat{A} q=\{\widehat{a} q, a \in A\}$ is a subset in $\partial P(\Sigma)$. Let us give some explanation how to understand what will be the set of a limit points. Let $\{g\}_{i \in \mathbb{N}}$ be a sequence of elements from $A$, then $g_{i}=g_{1}^{n_{i}} g_{2}^{m_{i}}$. Since we are looking for a limit points of the set $\left\{\widehat{g}_{i} q\right\}_{i \in \mathbb{N}}$ in the projective space we can assume that $g=\operatorname{diag}\left\{\alpha^{n_{i}+m_{i}}, \alpha^{-n_{i}+2 m_{i}}, 1\right\}$. Assume that $n_{i}>0, m_{i}>0$. Let $k$ be an integer such that $m_{i}=2 n_{i}+k_{i}$. There are three possibilities : $k_{i} \rightarrow \infty(1), k_{i} \rightarrow-\infty(2)$ and $k_{i} \rightarrow \kappa$, (3) when $i \rightarrow \infty$. Then the sequence of projective transformations $\widehat{g}_{i}$ converge to the quasi projective transformation (see the definition in H.Abels in this volume) $\widetilde{g}_{1}=\operatorname{diag}\{1,0,0\}$ in the case (1), $\widetilde{g}_{2}=\operatorname{diag}\{0,1,0\}$ in the case (2) and $\widetilde{g}_{3}=\operatorname{diag}\left\{\alpha^{k}, 1,0\right\}$ in the case (3). Conceder this maps as a projections $\pi_{i}, i=1,2,3$ of the space $\mathbb{R}^{3}$. Then the sequence $\left\{\widehat{g}_{i} q\right\}_{i \in \mathbb{N}}$ can converge to the points $\pi_{i} q, i=1,2,3$. It is not difficult to see that for every $\varepsilon$ there exists $N=$ $N(\varepsilon)$ such that if $a \in A_{N}$ then $d(\widehat{a} q, \partial L) \leq \varepsilon$ and if $U_{1}$ (respectively $U_{2}, U_{3}$ be a neighborhood of the point $\widehat{e_{1}}$, (respectively $\left.\widehat{e}_{2}, \widehat{e}_{3}\right)$ then the set $\partial L \backslash\left(U_{1} \cup U_{2} \cup\right.$ $\left.U_{3}\right)$ is finite.

We will say that two elements $g \in \Omega_{0}(G L(V))$ and $h \in \Omega_{0}(G L(V))$ are transversal if $A(g) \cup A\left(g^{-1}\right) \subseteq P \backslash C r(h)$ and $A(h) \cup A\left(h^{-1}\right) \subseteq P \backslash C r(g)$. The simple consequence of Lemma 1.4 is the following

Lemma 1.5. Let $S=\left\{g_{1}, \ldots, g_{m}\right\}$ be a subset of $\Omega_{0}(G L(V))$ such that $g_{i}$ and $g_{j}$

are transversal for every $i, j 1 \leq i, j \leq m$. Then there exists a positive integer $N$ such that for every sequence of positive integers $k_{1}, \ldots, k_{m}$, such that $k_{t} \geq N$ for all $\quad t \quad$ with $1 \leq t \leq m$ the set $S(k)=\left\{g_{1}^{k_{1}}, \ldots g_{m}^{k_{m}}\right\}$ is freely generates of the free group $\langle S(k)\rangle$.

Definition 1.6. Let $G$ be a subgroup of $G L(V)$ and $g_{0} \in \Omega_{0}(G)$. We say that

the set $F=\left\{g_{i} \in G, i \in I\right\}$ is a $g_{0}$-free system for $G$ (or simply $g_{0}$-free system if it is clear which group is considered) if $g_{i} \in \Omega_{0}(G)$ for all $i \in I$ and there exists a set of open subsets $O=\left\{O_{i}=O_{i}(F), O_{i} \subseteq P, i \in I\right\}$, a set of disjoint compact sets $K=\left\{K_{i}=K_{i}(F), K_{i} \subseteq P, i \in I\right\}$, an open $U_{0}=U\left(g_{0}\right)$ and a compact subset $K_{0}$ of $P$ such that

1. $A\left(g_{i}\right) \cup A\left(g_{i}^{-1}\right) \subseteq O_{i} \subseteq K_{i}$ for all $i \in I$,

2. $A\left(g_{0}\right) \cup A\left(g_{0}^{-1}\right) \subseteq U_{0} \subseteq K$

3. $\inf _{i \in I} d\left(K_{i}, C r\left(g_{0}\right)\right)>0$,

4, $K \subseteq P \backslash \overline{\cup_{i \in I} K_{i}}$

5. $\widehat{g}_{i}^{n} K_{j} \subseteq O_{i}$ for every $i, j \in I, i \neq j$ and non-zero $z \in \mathbb{Z}$.

6. $\widehat{g}_{i}^{n} K \subseteq O_{i}$ for every $i \in I$ and non-zero $z \in \mathbb{Z}$. 
From this definition immediately follows that the group generated by a $g_{0}$-free system $F$ is free and elements of $F$ are free generators.

Lemma 1.7. Let a finite subset $F$ of $G$ be a $g_{0}$-free system. Let $g$ be an element from $\Omega$ transversal to $g_{0}$ and to every element from $F$. Then there are two positive integers $N$ such that for every $n>N$ there exists a positive integer $M=M(n)$ such that if $\widehat{g}=g_{0}^{n} g^{m} g_{0}^{-n}$, then $\widetilde{F}=F \cup \widetilde{g}$ is a $g_{0}$-free system for all $m>M$.

Proof. Let $d_{1}=d\left(A^{+}(g) \cup A^{-}(g), C r\left(g_{0}\right)\right)$ and let $d_{2}=\inf _{i \in I} d\left(K_{i}, C r\left(g_{0}\right)\right)$. Put $d_{0}=1 / 4 \min \left(d_{1}, d_{2}\right)$ and let $B\left(A^{-}\left(g_{0}, d_{0}\right)\right)$ be a ball of radius $d_{0}$ with center in $A^{-}\left(g_{0}\right)$. It follows from Lemma 1.4 that there exists a positive integer $N_{1}=N_{1}\left(d_{0}, B\left(A^{-}\left(g_{0}\right), d_{0}\right)\right)$ such that ${\widehat{g_{0}}}^{-n} K_{i} \subseteq B\left(A^{-}\left(g_{0}\right), d_{0}\right)$ for all positive $n>N_{1}$. Since $A^{+}\left(x g x^{-1}\right)=\widehat{x} A^{+}(g), A^{-}\left(x g x^{-1}\right)=\widehat{x} A^{+}(g)$ and $C r\left(x g x^{-1}\right)=\widehat{x} C r(g)$, then $\overline{\cup_{i \in I} K_{i}} \subseteq P \backslash C r\left(g_{0}^{n} g g_{0}^{-n}\right)$ for all $n>N_{1}$. Set $d(n)=$ $d\left(\overline{\cup_{i \in I} K_{i}}, C r\left(g_{0}^{n} g g_{0}^{-n}\right)\right)$. By Lemma 1.4 there exists a positive integer $N_{2}=$ $N_{2}\left(d_{0}, U_{0}\right)$ such that $\widehat{g}_{0}^{n}\left(A^{+}(g) \cup A^{-}(g)\right) \subseteq U_{0}$ for all $n>N_{2}$. Two elements $g$ and $g_{0}$ are transversal, therefore two elements $g_{0}^{n} g g_{0}^{-n}$ and $g_{0}$ are transversal for all $n \geq \max \left\{N_{1}, N_{2}\right\}$. For $n \geq N$ set $h=g_{0}^{n} g g_{0}^{-n}$ and $d_{h}=d(n)$. There exists a compact subset $K_{h}$, an open subset $O_{h}$ in $P$ such that $A^{+}(h) \cap A^{-}(h) \subseteq O_{h} \subseteq K_{h} \subset$ $U_{0} \quad$ and $K_{h} \subseteq P \backslash C r\left(g_{0}\right)$. From $K_{h} \subseteq U_{0}$ follows that $\widehat{g}_{i}^{n} K_{h} \subseteq O_{i}$ for all $i \in I$. It follows from Lemma 1.4 that there exists $M_{1}=M_{1}\left(d_{h}\right)$ such that $\widehat{h}^{m} K_{i} \subseteq O_{h}$ for all $m,|m| \geq M_{1}$. Since $K_{h} \subseteq P \backslash C r\left(g_{0}\right)$ there exists a compact set $K^{*} \subset U_{0}$ such that $A^{+}(g) \cup A^{-}(g) \subseteq K^{*}$. By Lemma 1.4 there exists a positive integer $M_{2}$ such that $\widehat{h}^{m} K^{*} \subseteq O_{h}$ for all all integers $m,|m| \geq M_{2}$. Put $M=\max \left\{M_{1}, M_{2}\right\}$. Set $\widetilde{g}=$ $h^{m}$ then $\widetilde{F}=F \cup\{\widetilde{g}\}$ is a $g_{0}$-free system for $m \geq M$.

Then from Definition 1.6 no.5 follows that for every open subset $U$, ${\widehat{g_{0}}}^{n}\left(A^{+}(g) \cup A^{-}(g)\right) \subseteq U \subseteq U_{0}$ and $n>N_{2}$ for every $i \in I$ and positive integer $m$ we have $\widehat{g}_{i}^{m} U \subseteq O_{i}$. Lemma 1.4 now shows that there exists $M=$ $M(d(n), U)$ such that $\widehat{g}^{m}\left(K_{i}\right) \subseteq U$ for all $i \in I$ and $m>M$. Since elements $g$ and $g_{0}$ are transversal elements $g_{0}$ and $g_{0}^{m} g^{n} g_{0}^{-m}$ are transversal for all integers $m$ and $n$. Combining the above arguments, we conclude that $\widetilde{F}=F \cup \widetilde{g}$ is a $g_{0}$-free system.

Therefore we have the following important

Corollary 1.8. Let $F, g_{0}, g$ be as in Lemma 1.7. Let $H$ be a subgroup of finite index in $G$. Assume that $x \in G$ and $g \in x H$. Then there are two infinite sets of positive integers $N$ and $M$ such that $\widetilde{g}=g_{0}^{n} g^{m} g_{0}^{-n} \in x H$ and the set $\widetilde{F}=F \cup \widetilde{g}$ is a $g_{0}$-free system for all $n \in N, m \in M$.

The proof is straightforward. 
Assume now that :

1. There exists a proximal element in $G$.

2. The Zariski closure $\mathbb{G}$ of $G$ is a semisimple group.

3. $\mathbb{G}^{0} \cap G$ is an absolutely irreducible subgroup of $G L(V)$.

Then

Proposition 1.9. Let $H$ be a subgroup of $G$. Assume that $H$ contains a proximal element and $\mathbb{G}^{0} \cap H$ is Zariski dense in $\mathbb{G}^{0}$. Then for every $g \in G$ the set $g H \cap \Omega_{0}(G)$ is nonempty.

Remark 1.10. This important proposition first was proved in [MS 3]. A

different proof of this proposition can be deduced from the main theorem in [AMS].

The principal significance of the next theorem is in reduction to linear groups over local fields which allows us to use all above arguments. First this reduction was done in $[\mathrm{T}]$ for a finitely generated linear group where the Zariski closure is connected. A reduction to linear groups over local field in the general case when the Zariski closure is not necessarily connected is considerably more complicated. Theorem 1.10. Let $G$ be a finitely generated non virtually solvable linear group, than there exist a local field $k$ vector space $W$ over $k$ and irreducible representation $\rho: G \longrightarrow G L(W)$ such that

1. There exists a proximal element in $\rho(G)$.

2. The Zariski closure $\mathbb{G}$ of $\rho(G)$ is a semisimple group.

3. $\mathbb{G}^{0} \cap \rho(G)$ is an absolutely irreducible subgroup of $G L(W)$.

Since $G$ is not virtually solvable, we can assume that the Zariski closure of $G$ is semisimple. The proof splits naturally into a few steps.

Since we can reduce our group by taking a factor-group by non-trivial connected normal subgroup, it is easy to see that

Step 1. It is enough to prove our statement under the following assumption: the Zariski closure of $G$ is the wreath product $F$ ? $G_{*}$ where $G_{*}$ is a simple (nonconnected) algebraic group of an adjoint type and $F$ is a finite group. The descrip$\begin{array}{llllll}\text { tion } & \text { of } & F & G_{*} & \text { follows }\end{array}$ $F \curlyvee G_{*}=G_{*}^{F} \rtimes F$ where $F$ acts on $G_{*}^{F}$ by shift.

Step 2. Observe that it is enough to prove the statement of Theorem 1.10 for a group $G$ such that the Zariski closure of $G$ is a simple algebraic (not necessarily connected) group of adjoint type

Indeed by step 1 we can assume that our group is a wreath product $F$ ₹ $G_{*}$ where $G_{*}$ is a simple (non-connected) algebraic group of an adjoint type and $F$ is a 
finite group. In [AMS1, Theorem 5.17] we proved that if $G$ is a direct product $G=\Pi_{1}^{m} G_{i}$ of semisimple groups such for every $1 \leq i \leq m$ there exists $g^{(i)} \in G$ such the projection $\pi_{i}\left(g^{(i)}\right)$ of $g^{(i)}$ is a proximal element in $G_{i}$ then there exists an element $g \in G$ such that $\pi_{i}(g)$ is a proximal element for every $1 \leq i \leq m$. Hence if there exists a representation we need for $G_{*}$ taking the $m$-th tensor product of this representation we have an irreducible representation of $G^{F}$ which we can and will extend it to $F \nmid G_{*}$. This representation has all the necessary properties.

Since the Zariski closure $\mathbb{G}$ is a simple group of adjoint type we will assume that $\mathbb{G} \leq$ Aut $\mathbb{G}^{0}$

Step 3. This step splits naturally into two cases:

Case1. The factor group $\mathbb{G} / \mathbb{G}^{0}$ is cyclic.

Case 2. The factor group $\mathbb{G} / \mathbb{G}^{0}$ is the symmetric group of degree 3

Since the proof of the statement in the case 2 may be handle in much the same way as in case 1 , for the sake of exposition we restrict ourselves to the case $\mathbb{G} / \mathbb{G}^{0}$ is a cyclic group. By using standard arguments, we conclude that there exists a non torsion element $g \in G$ such that $\mathbb{G}$ is generated by $\mathbb{G}^{0} \cup\{g\}$. Since $G$ is finitely generated, it follows from [T, Lemma 4.1] that there exists a local field $k$ with absolute value $|\cdot|$ vector space $W$ over $k$ and absolutely irreducible representation $\rho: G \longrightarrow G L(W)$ such that $\rho(g)$ is proximal. We claim that the group $\rho\left(\mathbb{G}^{0} \cap\right.$ $G)=G_{1}$ is absolutely irreducible. Suppose the contrary. Let $\widetilde{W}=W \otimes_{k} \widetilde{k}$ and $W_{0}$ be a minimal $G_{1}$ invariant proper subspace of $W$. Put $x=\rho(g)$. Then there exist integers $i_{1}, \ldots, i_{t}$ such that $\widetilde{W}=W_{0} \oplus W_{i_{1}} \oplus \cdots \oplus W_{i_{t}}$ where $W_{i_{s}}=x^{i_{s}} W_{0}$. Since $x^{n}$ is a proximal element for all positive integer $n \in \mathbb{Z}$, the eigenvector $v$ corresponding to the maximal eigenvalue belongs to some $W_{i}$. Suppose for instance $v \in W_{0}$. Then $x^{i_{1}} v \in W_{i_{1}}$. Therefore $x^{i_{1}} v$ and $v$ are two different eigenvectors for a proximal element $x$ and correspond to the maximal eigenvalue, a contradiction. Remark 1.12. In the reduction done in $[\mathrm{T}]$, Tits used an easy but crucial lemma

saying that for a finitely generated infinite group $G$ there exists a local field $k$ with a absolute value $|\cdot|$ such that for some element $g \in G$ at least one eigenvalue has an absolute value $\neq 1$. This fact can be deduced from a natural and useful generalization proved in [BG2, Lemma 2.1].

\section{Dense free subgroups.}


For a start let us prove the Platonov problem.

Proof Observe that there exists a proximal element in $S L_{n}(\mathbb{Z})$. Indeed, let $g$ be

a diagonal matrix $g=\operatorname{diag}\left(a_{1}, \ldots, a_{n}\right), a_{i}>0 a_{i} \in \mathbb{R}, a_{1}>a_{2} \geq \cdots \geq a_{n}$. Since the action of the group generated by $g$ on $S L_{n}(\mathbb{R}) / S L_{n}(\mathbb{Z})$ is ergodic, then by well-known arguments $[\mathrm{R}]$ for every neighborhood of identity $U \in S L_{n}(\mathbb{R})$ there exists an infinite set of positive integers $M$ such that the intersection $U g^{m} U \cap$ $S L_{n}(\mathbb{Z}) \neq \emptyset, m \in M$. From $g \in \Omega_{0}\left(S L_{n}(\mathbb{R})\right)$ follows that there are a positive integer $M$ and a neighborhood of identity $U, U \subseteq S L_{n}(\mathbb{R})$ such that $U g^{m} U \subseteq$ $\Omega_{0}\left(S L_{n}(\mathbb{R})\right)$ for $m \geq M$. Let $g_{0} \in \Omega_{0}\left(S L_{n}(\mathbb{R})\right) \bigcap S L_{n}(\mathbb{Z})$. The group $S L_{n}(\mathbb{Z})$ is absolutely irreducible; therefore there exists $x \in S L_{n}(\mathbb{Z})$ such that two elements $h=x g_{0} x^{-1}$ and $g_{0}$ are transversal. Consequently, there exists a positive integer $k$ such that $\left\{h^{k}\right\}$ is a $g_{0}$ free system. Therefore we will assume that $\{h\}$ is a $g_{0}$-free system. It is not difficult to show that there exists a $g_{0}$-free system $F=\left\{h_{1}, \ldots, h_{s}\right\}$ such that the group generated by $F$ is Zariski dense in $S L_{n}(\mathbb{R})$. Then $[\mathrm{W}]$ for $n \geq 3$ the pro- finite closure $F^{*}$ of $F$ is subgroup of finite index in $S L_{n}(\mathbb{Z})$. Let $x_{i} F^{*}, i=1, \ldots, t$ be all different classes $S L_{n}(\mathbb{Z}) / F^{*}$. By Corollary 1.8, there exist elements $h_{s+1}, h_{s+t}$ such that $h_{s+i} \in x_{i} F^{*}, i=1, \ldots, t$ and $F_{0}=\left\{h_{1}, \ldots, h_{s}, h_{s+1}, \ldots, h_{s+t}\right\}$ is a $g_{0}$-free system. It is clear that the group generated by $F_{0}$ is pro-finitely dense in $S L_{n}(\mathbb{Z})$ for $n \geq 3$. Since the group $S L_{n}(\mathbb{Z})$ is finitely generated there exists a maximal proper subgroup $H$ which contains $F_{0}$. Obviously $\mathrm{H}$ is a maximal subgroup of $S L_{n}(\mathbb{Z})$. Assume that the index $S L_{n}(\mathbb{Z}) / H$ is finite. Then $H$ is a proper pro-finitely dense open subgroup of $S L_{n}(\mathbb{Z})$ which is impossible and the proof is completed.

Since a subgroup of a finite index of a finitely generated group is a finitely generated group we reformulated [MS1] Platonov's problem as following:

Conjecture (G. Margulis, G. Soifer) Let $G=S L_{n}(\mathbb{Z})$ and $H$ be a maximal subgroup of $G$. Assume that $H$ is a finitely generated group, then the index $G / H$ is finite.

This conjecture is true for $n=2$. Furthermore it is true if $G$ is a lattice in $S L_{2}(\mathbb{R})$ (see [SV]). Y. Glasner pointed out that $S L_{n}(\mathbb{Z})$ is a maximal subgroup of infinite index of a lattice $S L_{n}(\mathbb{Z}[1 / p])$. Nevertheless the above conjecture is still open for $n \geq 3$.

When our results were announced [MS 1] we received a letter from G.Prasad with the following conjecture

Conjecture (G. Prasad, J. Tits) Every maximal subgroup of $S L_{n}(\mathbb{Z}), n \geq 3$ of infinite index is virtually free.

We show in [MS2] that there exists a not virtually free maximal subgroup of an infinite index in $S L_{n}(\mathbb{Z})$ for $n \geq 4$. Now we will show that for $n=3$ this conjecture is also not true. Recall that the group $S L_{2}(\mathbb{Z})$ is virtually free. 
Theorem 2.1. There exists a maximal subgroup of infinite index in $S L_{3}(\mathbb{Z})$ which is not virtually free.

Proof. Let $G=S L_{3}(\mathbb{R})$ and $\Gamma=S L_{3}(\mathbb{Z})$. Let $g_{1}, g_{2}$ be two commuting elements from $\Omega(\Gamma)$ which generate a free abelian group of rank 2 . Let $e_{1}, e_{2}, e_{3}$ be their eigenvectors corresponding to a three different eigenvalues. Consider the set $\Sigma=$ $\left\{t_{1} e_{1}+t_{2} e_{2}+t_{3} e_{3}, \sum_{1}^{3} t_{i}=1, t_{i} \geq 0, i=1,2,3\right\}$. Let $P(\Sigma)$ be the projectivization of $\Sigma$ and let $\partial P(\Sigma)$ be the boundary of $P(\Sigma)$. Let $q$ (correspondingly, $\widehat{e}_{i} i=$ $1,2,3)$ be the point of $P(\Sigma)$ which corresponds to the line span by the vector $1 / 3 e_{1}+1 / 3 e_{2}+1 / 3 e_{3}$ (correspondingly, $e_{i} i=1,2,3$ ). Analysis similar to that in Example 1 with a bit more routine calculations shows that elements $g_{1}$ and $g_{2}$ will fulfil following properties:

1. The boundary $\partial L$ of the orbit $L=\widehat{A} q=\{\widehat{a} q, a \in A\}$ is a subset of $\partial P(\Sigma)$

2. Let $U_{i}$ be a neighborhood of the point $\widehat{e_{i}}, i=1,2,3$. Then the set $\partial L \backslash\left(U_{1} \cup\right.$ $\left.U_{2} \cup U_{3}\right)$ is finite.

Hence there are two lines $L_{1}$ and $L_{2}$ in the projective space $P$ such that $\partial L \cap$ $L_{i}=\emptyset$ for $i=1,2$ and $q=L_{1} \cap L_{2}$. It is easy to see that there exists a positive integer $N$ and neighborhood $W$ of the point $q$ and a compact $K_{0}, W \subseteq K_{0}$ such that $\widehat{a}^{n} K_{0} \cap\left(L_{1} \cup L_{2}\right)=\emptyset$ for all $a \in A$ and $|n| \geq N$. Let $g_{0}$ be a hyperbolic element of $G$ such that $B^{+}\left(g_{0}\right)=L_{1}, B^{-}\left(g_{0}\right)=L_{2}$ and $A^{+}\left(g_{0}\right) \cup A^{-}\left(g_{0}\right) \subseteq W$. Put $A_{0}=<g_{1}^{N}, g_{2}^{N}>$. Then there exists a positive $d$ such that the distance $\min _{a \in A_{0}} d\left(\widehat{a} K_{0}, L_{1}\right), d\left(\widehat{a} K_{0}, L_{2}\right)>d$. Since the action of the subgroup group generated by $g_{0}$ on $S L_{3}(\mathbb{R}) / S L_{3}(\mathbb{Z})$ is ergodic, for every neighborhood $U$ of the identity in the group $G$ there exists an infinite set of positive integers $M$ such that $U g_{0}^{r} U \cap \Gamma \neq \emptyset$. Hence because for $m \in M A^{+}\left(g^{m}\right)=A^{+}(g), A^{-}\left(g^{m}\right)=$ $A^{-}(g), B^{+}\left(g^{m}\right)=B^{+}(g)$ and $B^{-}\left(g^{m}\right)=B^{-}(g)$ for every positive $\varepsilon$ there exist neighborhood $U$ of the identity in the group $G$ and positive integer $M_{0}$ such that if $m \geq M_{0}$ for every $g \in U g_{0}^{r} U$ we have

1. $g \in \Omega_{0}(G)$,

2. $A^{+}(g) \cup A^{-}(g) \subseteq W$,

3. $\overline{\cup_{n \in \mathbb{Z}} \widehat{a}^{n} K_{0}} \subseteq P \backslash\left(B^{+}(g) \cup B^{-}(g)\right)$.

Therefore we can and will assume that $g_{0} \in \Gamma$. Let $g$ be an element from $\Gamma$ transversal to $g_{0}$. It follows from (2) and (3) since $A^{+}\left(g_{0}\right) \cup A^{-}\left(g_{0}\right) \subseteq P \backslash\left(B^{+}(g) \cup\right.$ $\left.B^{-}(g)\right)$, that there exists a positive integer $N_{0}$ such that for $n \geq N_{0}$ we have $g_{0}^{-n} \frac{\cup_{n \in \mathbb{Z}} \widehat{a}^{n} K_{0}}{} g(n)_{0} \subseteq P \backslash\left(B^{+}(g) \cup B^{-}(g)\right)$. Hence there exists a positive integer $N_{0}$ such that for $n \geq N_{0}$ we have $B^{+}\left(g_{0}^{n} g g_{0}^{-n}\right) \cup B^{-}\left(g_{0}^{n} g g_{0}^{-n}\right) \subseteq P \backslash \overline{\cup_{n \in \mathbb{Z}} \widehat{a}^{n} K_{0}}$. Repeated application of Corollary 1.8 enables us using an arguments from [SV] to claim that there exists a finite $g_{0}$-free system $F=\left\{f_{i}, 1 \leq i \leq m\right\}$ such that 
1. The group generated by $F$ is pro-finitely dense in $\Gamma$.

2. $A^{+}\left(f_{i}\right) \cup A^{-}\left(f_{i}\right) \subseteq W$ for every $i, 1 \leq i \leq m$.

3. $d\left(\widehat{a}^{n} K_{0},\left(B^{+}\left(f_{i}\right) \cup B^{-}\left(f_{i}\right)\right)>d / 2\right.$ for every $1 \leq i \leq m$ and $a \in A_{0}$.

Indeed, assume that $f \in \Omega(\Gamma)$ is an element transversal to $g_{0}$. Since for every neighborhood $U$ of $A^{-}\left(g_{0}\right)$ there exists a positive integer $N_{0}$ such that for all $n \geq$ $N_{0}$ we have $\widehat{g}^{-n_{0}}\left(\overline{\cup_{n \in \mathbb{Z}} \widehat{a}^{n} K_{0}}\right) \subseteq U$. Therefore if $U \in P \backslash\left(B^{+}(f) \cup B^{-}(f)\right)$ then $\widehat{g}^{-n_{0}}\left(\overline{\cup_{n \in \mathbb{Z}} \widehat{a}^{n} K_{0}}\right) \subseteq P \backslash\left(B^{+}(f) \cup B^{-}(f)\right)$ for $n \geq N_{0}$. Hence $\overline{\cup_{n \in \mathbb{Z}} \widehat{a}^{n} K_{0}} \subseteq P \backslash$ $\left(B^{+}\left(g^{n} f g^{-n}\right) \cup B^{-}\left(g^{n} f g^{-n}\right)\right)$ for $n \geq N_{0}$. As we proved in [SV] there exists a finite set of elements $f_{1}, \ldots, f_{k}$ such that the Zariski closure of the group $<f_{s}>$ is connected for every 1 leqs $\leq k$ and the pro-finite completion of the group $F_{1}=<$ $f_{1}, \ldots, f_{k}>$ is a subgroup of a finite index in $\Gamma$. It follows from Lemma 1.7 that we can and will assume that $f_{1}, \ldots, f_{k}$ is a $g_{0}$-free system and fulfil property no3. Let $T_{1}, ; T_{r}$ be all different co-sets $\Gamma / F_{1}$. Repeated arguments above enabled us to show that there are elements $f_{k+1}, \ldots, f_{k+r}$ such that the set $f_{1}, \ldots f_{k+r}$ is a $g_{0}$-free system which fulfil properties no1,2,3.

It follows from no. 3 that there exists a compact $K_{N}$ such that $\cup_{n \in \mathbb{Z}} \widehat{a}^{n} K_{0} \subseteq K_{N}$ and $d\left(K_{N}, B^{+}\left(f_{i}\right) \cup B^{-}\left(f_{i}\right)\right)>d / 4$. It follows from Lemma 1.4 that there exists a positive number $N_{1}$ such that $f_{i}^{n} K_{N} \subseteq W$ for all $n \geq N_{1}$. Set $n_{i}=2 N_{1}$ for $i=1, \ldots, k$ and $n_{i} \geq N_{1}, f_{i}^{n_{i}} \in M_{i}$ for $i=k+1, \ldots, k+r$. Then the group $F=<f_{1}^{n_{1}}, \ldots f_{k+r}^{n_{k+r}}>$ is a free subgroup which is pro-finitely dense in $\Gamma$ such that $\widehat{f} K_{N} \subseteq W$ for every $f \in F$. Hence the group generated by $A_{0}$ and $F$ is a free product $A_{0} * F$. Therefore a maximal subgroup of $\Gamma$ which contains the group generated by $A_{0}$ and $\widetilde{F}$ will be a maximal subgroup of $\Gamma$. This subgroup will be of infinite index since the group generated by $\widetilde{F}$ is pro-finitely dense. This subgroup contains a free abelian group $A_{0}$ of rank 2 . Hence it is not virtually free.

There are some other results in the spirit of the statement of Theorem 2.1, see for example $[\mathrm{S}],[\mathrm{V}]$.

Let $G$ be a subgroup of $G L_{n}(k)$ where $k$ is a local field. The full linear group $G L_{n}(k)$ and hence any subgroup of it is endowed with the standard topology that is the topology induced from the local field $k$. We will denote by $\|\cdot\|$ a norm on $G L_{n}(k)$ induced from the local field absolute value $|\cdot|$. Let $S=\left\{s_{1}, \ldots, s_{m}, s_{i} \in\right.$ $\left.G L_{n}(k)\right\}$ be a finite set. Put $S(\varepsilon)=\left\{\left(\widehat{s}_{1}, \ldots, \widehat{s}_{m}\right)\right.$ such that $\left\|\widehat{s}_{i}-s_{i}\right\| \leq \varepsilon$ for all $1 \leq i \leq m$ and $\varepsilon>0\}$. Assume that $\Gamma$ is a finitely generated dense subgroup of a connected semisimple group $G, G \leq G L_{n}(k)$. We claim that there exists $\varepsilon_{0}$ such that for every $\varepsilon<\varepsilon_{0}$ the group generated by the set $\left\{\widehat{s_{1}}, \ldots, \widehat{s_{m}}\right\}$ where $\left(\widehat{s_{1}}, \ldots, \widehat{s_{m}}\right) \in S(\varepsilon)$ is dense in $G$ (see [BG2, 5.1]). Indeed, there exist a finite set $S_{1}=\left\{g_{1}, \ldots, g_{l}\right\}$ where $l=\operatorname{dim} G$ and $\varepsilon_{1}$ such that if $\left\|\widehat{g}_{i}-g_{i}\right\| \leq \varepsilon_{1}$ for $i=$ $1, \ldots, l$ then the group generated by $\widehat{S}_{1}=\left\{\widehat{g}_{1}, \ldots, \widehat{g}_{l}\right\}$ is dense in $G$. Since $\Gamma$ is dense in $G$ then there exist elements $\widehat{\gamma}_{i} \in \Gamma$ such that $\left\|\widehat{\gamma}_{i}-g_{i}\right\| \leq \varepsilon_{1} / 2$ for all $i=$ 
$1, \ldots, l$. Then $\widehat{\gamma}_{i}=w_{i}\left(s_{1}, \ldots, s_{m}\right)$. Consider maps $w_{i}: \underbrace{G \times \cdots \times G}_{m} \longrightarrow G$ where $i=1, \ldots, l$. There exists an $\varepsilon$ such that if $\left\|\widehat{s}_{i}-s_{i}\right\| \leq \varepsilon$ for all $i=1, \ldots, l$ then $\left\|w_{i}\left(\widehat{s}_{1}, \ldots, \widehat{s}_{m}\right)-w_{i}\left(s_{1}, \ldots, s_{m}\right)\right\| \leq \varepsilon_{1}$ and therefore $\left\|w_{i}\left(\widehat{s}_{1}, \ldots, \widehat{s}_{m}\right)-g_{i}\right\| \leq \varepsilon_{1}$ for all $i=1, \ldots, l$. Hence the group generated by the set $\left\{\widehat{s}_{1}, \ldots, \widehat{s}_{m}\right\}$ will be dense in $G$.

E. Breuillard and T. Gelander proved in [BG2] the following topological Tits alternative.

Theorem 2.2 [BG2]. Let $k$ be a local field and $\Gamma$ a subgroup of $G L_{n}(k)$. Then $\Gamma$ contains either open solvable subgroup or a dense free subgroup.

Note that for a non discrete subgroup $\Gamma$ the two cases are mutually exclusive. Hence if $\Gamma$ a dense subgroup of a semisimple connected Lie group $G$, then for any set

$S=\left\{\gamma_{1}, \ldots, \gamma_{m}\right\}$ of generators of $\Gamma$ there exists an $\varepsilon_{0}$ such that for every $\varepsilon \leq \varepsilon_{0}$ there exists $\widehat{S}=\left(\widehat{\gamma}_{1}, \ldots, \widehat{\gamma}_{m}\right) \in S(\varepsilon) \cap \underbrace{\Gamma \times \cdots \times \Gamma}_{m}$ such that the group $<\widehat{S}>$ is free and dense in $G$. For a compact connected Lie group one can deduce this fact for a from [S, Proposition 4.5].

Let $G$ be a connected compact group Lie. Then the set of torsion elements of $G$ is dense in $G$. Therefore for every $\varepsilon$ and any set of generators $S=\left\{\gamma_{1}, \ldots, \gamma_{m}\right\}$ of a subgroup $\Gamma$ there exists $\left(\widehat{\gamma}_{1}, \ldots, \widehat{\gamma}_{m}\right) \in S(\varepsilon)$ such that the group generated by $\widehat{S}=\left\{\widehat{\gamma}_{1}, \ldots, \widehat{\gamma}_{m}\right\}$ is not free because it contains torsion. On the other hand every finitely generated linear group has a subgroup of a finite index without torsion. Therefore we state the following

Conjecture. (G. Margulis, G. Soifer.) Let $G$ be a non solvable connected Lie group. Assume that the subgroup of $G$ generated by a set $S=\left\{s_{1}, \ldots, s_{m}\right\}$ is a free dense subgroup. Then for every $\varepsilon$ there exists $\left(s_{1}^{*}, \ldots, s_{m}^{*}\right) \in S(\varepsilon)$ such that the group generated by the set $S^{*}=\left\{s_{1}^{*}, \ldots, s_{m}^{*}\right\}$ is not virtually free.

This conjecture first was stated for a compact group and it was proved recently by T.Gelander. His results show that it will interesting to answer to the following Problem. Let $G$ be a connected Lie group let $F$ be a free dense subgroup of $G$ generated by a set $S=\left\{s_{1}, \ldots s_{n}\right\}$. Is it true that for every dense subgroup $\Gamma$ of $G$ and every $\varepsilon$ there exists a set $\left\{\gamma_{1}, \ldots, \gamma_{n}\right\}$ such that $\left\{\gamma_{1}, \ldots, \gamma_{n}\right\} \subseteq S(\varepsilon)$ and $\left.<\gamma_{1}, \ldots, \gamma_{n}\right\rangle=\Gamma$.

\section{Euclidean crystallographic semigroups.}


Recall that a semigroup $S$ acts properly discontinuously on a topological space $X$ if for every compact subset $K \subseteq X$ the set $\{s \in S \mid s K \cap K \neq \varnothing\}$ is finite. A semigroup $S$ is called crystallographic if it acts properly discontinuously and there exists a compact subset $K_{0} \subseteq X$ such that $\cup_{s \in S} s K_{0}=X$. In the case when $X=\mathbb{R}^{n}$ and $S \subseteq$ Isom $X, S$ is called a Euclidean crystallographic semigroup.

In this section we will prove the following conjecture due to G.Margulis.

Conjecture Let $S$ be an Euclidean crystallographic semigroup, then $S$ is a group.

Here is a scenario of our proof of the above conjecture. The main idea of the proof is to show that the Zariski closure $G$ of a semigroup $S$ does not contain a free subgroup. We prove this using our ideas and results from [S]. Therefore by the Tits' alternative $G$ is a virtually solvable group. Hence the linear part of $G$ is a compact virtually solvable group. Consequently the linear part of $G$ is virtually abelian. Combining this with the fact that $S$ acts properly discontinuously, we show that every element in $S$ is invertible. Thus $S$ is a group.

Let us recall some necessary definitions . Let $G=\operatorname{Aff} \mathbb{R}^{n}$ be the group of all affine transformation of the $n$-dimensional real affine space $\mathbb{R}^{n}$. This group is the semidirect product of $G L_{n}(\mathbb{R})$ and the subgroup of all parallel translations which can be identified with $\mathbb{R}^{n}$, i.e,

$$
\operatorname{Aff}^{n}=\mathbb{R}^{n} \rtimes \operatorname{GL}_{n}(\mathbb{R}) .
$$

We will consider the natural homomorphism

$$
\ell: \operatorname{Aff} \mathbb{R}^{\mathrm{n}} \longrightarrow \mathrm{GL}_{\mathrm{n}}(\mathbb{R}),
$$

and because the group Aff $\mathbb{R}^{\mathrm{n}}$ is semidirect product we have for every element $g \in$ Aff $\mathbb{R}^{\mathrm{n}}$ the decomposition

$$
g=v_{g} \ell(g), \quad v_{g} \in \mathbb{R}^{n}, \quad \ell(g) \in G L_{n}\left(\mathbb{R}^{n}\right) .
$$

Let $q$ be a positive definite quadratic form on $\mathbb{R}^{n}$. Then

$$
\text { Isom } \mathbb{R}^{\mathrm{n}}=\left\{\mathrm{g} \in \mathrm{Aff} \mathbb{R}^{\mathrm{n}}: \mathrm{q}(\ell(\mathrm{g})(\mathrm{x}))=\mathrm{q}(\mathrm{x})\right\} .
$$

Hence if $g \in \operatorname{Isom} \mathbb{R}^{\mathrm{n}}$ then $\ell(g) \in O(q)$. Let $g \in \operatorname{Isom} \mathbb{R}^{\mathrm{n}}$ and let $V^{0}(g)=\{v \in$ $\left.\mathbb{R}^{n}: \ell(g) v=v\right\}$. Recall that an element $g$ of an algebraic group $G, G \subseteq G L_{n}(\mathbb{R})$ is called regular if and only if

$$
\operatorname{dim} V^{0}(g)=\min _{x \in G} V^{0}(x) .
$$

Let $g \in$ Aff $\mathbb{R}^{\mathrm{n}}$ then there exists the maximal $g$-invariant affine space $A^{0}(g)$ of $\mathbb{R}^{n}$ such that $g$ induces a translation on it. This translation can be zero and in 
that case all points in $A^{0}(g)$ are fixed points. It is easy to see that if we define the vector $v^{0}(g)$ as follows: consider a point $x \in A^{0}(g)$ put

$$
v^{0}(g)=\frac{g x-x}{\|g x-x\|}
$$

then this vector does not depend on $x$. We define $v^{0}(g)=0$ if there exists a $g$-fixed point. For every set of vectors $X$ in $\mathbb{R}^{n}$ there is a smallest convex cone $C_{X}$ such that $X \subseteq C_{X}$. Therefore for every semigroup $S \subseteq$ Isom $\mathbb{R}^{\mathrm{n}}$ there exists the convex cone $C(S)$ defined as $C(S)=C_{X}$, where $X=\left\{v^{0}(g), g \in\right.$ $S, \ell(g)$ is a regular element of the group $\overline{\ell(\mathrm{S})}\}$. Let $V^{0}(S)$ be the subspace generated by the set $\left\{V^{0}(g)\right\}_{g \in S}$. Assume that there is no non-trivial $S$-invariant affine subspaces of the affine space $\mathbb{R}^{n}$ then

Lemma 3.1. $V^{0}(S)=\mathbb{R}^{n}$.

Proof. It immediately follows from [S, Lemma 4.1] that $V^{0}(S)$ is an $\ell(S)$-invariant subspace of the vector space $\mathbb{R}^{n}$. This subspace is non-trivial because $S$ acts properly discontinuously. Since the closure $\overline{\ell(S)}$ is a reductive group, there exists an $\ell(S)$-invariant subspace $W$ of $\mathbb{R}^{n}$ such that $V^{0}(S) \oplus W=\mathbb{R}^{n}$. Assume that $W \neq\{0\}$. We have the natural projection of the affine space $\mathbb{R}^{n}$ onto to the affine space $A_{1}=\mathbb{R}^{n} / V^{0}(S)$ along $V^{0}(S)$ and hence an induced homomorphism $\rho: S \rightarrow$ Isom $A_{1}$. Let $\ell(\rho(g))$ be the linear part of $\rho(g)$. Since $V^{0}(g) \subseteq V^{0}(S)$ there exists a fixed point for every element $\rho(g), g \in S$. Therefore the closure of the group generated by $\rho(g)$ is compact for every $g \in S$. Thus the group $\overline{\rho(S)}$ is compact. Hence there exists a $\overline{\rho(S)}$-fixed point $p_{0}$ in $A_{1}$. Consequently $p_{0}+V^{0}(S)$ is non-trivial proper $S$-invariant affine subspace. Contradiction which proves the lemma.

Consider the closure $\overline{C(S)}$ of the cone $C(S)$ in $\mathbb{R}^{n}$. Our next goal is to prove Lemma 3.2 Let $S$ be a crystallographic semigroup. Then $C(S)=\mathbb{R}^{n}$.

Proof. By $\left[\mathrm{S}\right.$, Lemma 4.1] it is enough to prove that $\overline{C(S)}=\mathbb{R}^{n}$. To obtain a contradiction assume that $\overline{C(S)} \neq \mathbb{R}^{n}$. Then there exists a non-zero vector $v_{0}$ in $\mathbb{R}^{n}$ such that the scalar product $\left(v, v_{0}\right) \leq 0$ for all $v \in C(S)$ and there is a vector $\widetilde{v} \in C(S)$ such that $\left(\widetilde{v}, v_{0}\right)<0$. It follows from our assumption and Lemma 3.1 that there exists an element $s_{0} \in S$ such that $V^{0}\left(s_{0}\right) \varsubsetneqq C(S)$. Let $v^{*}$ be a vector from $V^{0}\left(s_{0}\right)$ such that $\left(v^{*}, v_{0}\right)>0$.

Let $K$ be a compact subset in $\mathbb{R}^{n}$ such that $\cup_{s \in S} s K=\mathbb{R}^{n}$. Fix a point $p_{0}$ in $K$. It is clear that if $p_{m}$ is a point of $\mathbb{R}^{n}$, where $p_{m}=p_{0}+m v_{0}$ and $m$ is a positive number, then there exists $s_{m} \in S$ such $p_{m} \in s_{m} K$. Therefore for every point $p \in$ K

$$
\lim _{m \rightarrow \infty} \frac{s_{m} p-p}{\left\|s_{m} p-p\right\|}=v^{*} .
$$


Consider the subset $\left\{\ell\left(s_{m}\right)\right\}_{m \in \mathbb{N}}$ of the compact group $O(q)$. We can and will assume that this sequence is converge to some element $g_{0}$ of the closure $\overline{\ell(S)}$ in $O(q)$. Put $g_{1}=\ell\left(s_{0}\right) g_{0}^{-1}$. Since $g_{1} \in \overline{\ell(S)}$ there is a sequence $\left\{\bar{s}_{m}\right\}_{m \in \mathbb{N}}$ of elements from $S$ such that the sequence $\left\{\ell\left(\bar{s}_{m}\right)\right\}_{m \in \mathbb{N}}$ converges to $g_{1}$. We can assume that for every point $p \in K$ we have

$$
\lim _{m \rightarrow \infty} \frac{\left\|\bar{s}_{m}(p)-p\right\|}{\left\|s_{m} p-p\right\|}=0
$$

Define $t_{m}=\bar{s}_{m} s_{m}$ for all positive integers $m$. Let us show, that

$$
\lim _{m \rightarrow \infty}\left(v^{0}\left(t_{m}\right), v_{0}\right)>0 .
$$

Indeed, let $q_{m}=\bar{s}_{m}^{-1} p_{0}$ and let $v_{m}$ be a vector $v_{m}=t_{m} q_{m}-q_{m} /\left\|t_{m} q_{m}-q_{m}\right\|$. Since $t_{m} q_{m}-q_{m}=t_{m} p_{0}-p_{0}+\bar{s}_{m} q_{m}-q_{m}$ and $\left\|\bar{s}_{m} q_{m}-q_{m}\right\|=\left\|\bar{s}_{m} p_{0}-p_{0}\right\|$ from (3.2) follows that

$$
\lim _{m \rightarrow \infty} v_{m}=v^{*} .
$$

The sequence $\left\{\ell\left(\bar{s}_{m}\right)\right\}_{m \in \mathbb{N}}$ converges to $g_{1}$. Therefore the sequence $\left\{\ell\left(t_{m}\right)\right\}_{m \in \mathbb{N}}$ converges to $\ell\left(s_{0}\right)$. Thus the sequence $\left\{V^{0}\left(t_{m}\right)\right\}_{m \in \mathbb{N}}$ converges to the subspace $V^{0}(t)$. Now we use the following idea. Let $g$ be an euclidian transformation and let $\pi_{g}: \mathbb{R}^{n} \longrightarrow A^{0}(g)$ be the orthogonal projection onto $A^{0}(g)$. Then for every point $x$ of $\mathbb{R}^{n}$ we have $\pi_{g}(g x-x)=\alpha v^{0}(g)$ where $\alpha=\left\|\pi_{g}(g x-x)\right\|$. Consequently because the sequence $\left\{V^{0}\left(t_{m}\right)\right\}_{m \in \mathbb{N}}$ converges to the subspace $V^{0}(t)$ from (3.4) follows that $\lim _{m \rightarrow \infty} v_{m}^{0}=v^{*}$. Hence $\lim _{m \rightarrow \infty}\left(v^{0}\left(t_{m}\right), v_{0}\right)>0$.. On the other hand, $v^{0}\left(t_{m}\right) \in \overline{C(S)}$. Therefore $\left(v^{0}\left(t_{m}\right), v_{0}\right) \leq 0$. Contradiction which proves the lemma.

We will use the following fact proved in [S, Proposition 4.5].

Proposition 3.3. Let $S$ be a subsemigroup of Isom $\mathbb{R}^{n}$. Assume that $\overline{\ell(S)}$ is a connected non-solvable group. Then for every finite set $g_{1}, \ldots, g_{m} \subseteq S$ such that $v^{0}\left(g_{i}\right)$ non-zero for all $i, 1 \leq i \leq m$, and every positive $\varepsilon, \varepsilon<1, \varepsilon \in \mathbb{R}$, there are elements $g_{1}^{*}, \ldots, g_{m}^{*} \subseteq S$ such that

(1) Semigroup generated by $g_{1}^{*}, \ldots, g_{m}^{*}$ is free and $g_{1}^{*}, \ldots, g_{m}^{*}$ are free generators,

(2) $\left(v^{0}\left(g_{i}^{*}\right), v^{0}\left(g_{i}\right)\right)>1-\varepsilon$ for all $i, 1 \leq i \leq m$.

Proposition 3.4. Let $S$ be a crystallographic semigroup, then the Zariski closure $G$ of $S$ is a virtually solvable group.

Proof. On the contrary assume that the group $G$ is not virtually solvable. Without loss of generality we can assume that the group $G$ is connected. It follows from Lemma 3.1 and Lemma 3.2 that there exists a finite subset $\left\{s_{1}, \ldots, s_{m}\right\}$ of 
$S$ such that convex hole of the set $\left\{v^{0}\left(s_{1}\right), \ldots, v^{0}\left(s_{m}\right)\right\}$ is $\mathbb{R}^{n}$. Hence there exists a positive real number $\varepsilon, \varepsilon<1$ such that if vectors $w_{1}, \ldots, w_{m}$ are taken such that $\left(w_{i}, v^{0}\left(s_{i}\right)\right) \geq 1-\varepsilon$ then the convex hole of the set $\left\{w_{1}, \ldots, w_{m}\right\}$ is $\mathbb{R}^{n}$. Let $s_{0}$ be any element of $S$ such that $v^{0}\left(s_{0}\right) \neq 0$. It follows from Proposition 3.3 that there exists a subset $s_{0}^{*}, s_{1}^{*} \ldots, s_{m}^{*}$ such that

(1) subgroup generated by the subset $\left\{s_{0}^{*}, s_{1}^{*} \ldots, s_{m}^{*}\right\}$ is free and $s_{0}^{*}, s_{1}^{*} \ldots, s_{m}^{*}$ are free generators,

(2) convex hole of the set $\left\{v^{0}\left(s_{1}^{*}\right), \ldots, v^{0}\left(s_{m}^{*}\right)\right\}$ is $\mathbb{R}^{n}$.

Since the convex hole of the set $\left\{v^{0}\left(s_{1}^{*}\right), \ldots, v^{0}\left(s_{m}^{*}\right)\right\}$ is $\mathbb{R}^{n}$, there exists a positive real number $\delta=\delta\left(s_{1}^{*} \ldots, s_{m}^{*}\right)$ such that for arbitrary unite vector $v \in \mathbb{R}^{n}$ and some vector $v_{i_{0}}, 1 \leq i_{0} \leq m$ we have $\cos \measuredangle\left(v, v_{i_{0}}\right) \leq-\delta$. Let $x_{i} \in A^{0}\left(s_{i}^{*}\right)$ be a point $i, 1 \leq i \leq m$. Put $l_{i}=\left\|s_{i}^{*} x_{i}-x_{i}\right\|$ for each $i, 1 \leq i \leq m$. Set $l=$ $\max _{1 \leq i \leq m} l_{i}$. Fix a point $p_{0} \in \mathbb{R}^{n}$. Let $p$ be a point of $\mathbb{R}^{n}$. It is easy to see that there exist positive integers $j, 1 \leq j \leq m$ and $k_{j}$ such that $d\left(\left(s_{j}^{*}\right)^{k_{j}} p, p_{0}\right) \leq$ $\sqrt{1-\delta^{2}} d\left(p, p_{0}\right)+\frac{1}{2} l$. Assume that the euclidian distance $d\left(p, p_{0}\right)>\frac{2 l}{\delta^{2}}$. Then there is a positive number $\theta=\theta(\delta) \leq 1$ such that if $d\left(p, p_{0}\right) \geq \frac{2 l}{\delta^{2}}$ we have

$$
d\left(\left(s_{j}^{*}\right)^{m_{j}} p, p_{0}\right) \leq \theta d\left(p, p_{0}\right) .
$$

Let us now define the following infinite set of disjoint subsets $S_{j}$ of $S$. Let $S^{*}$ be a subsemigroup generated by the set $\left\{s_{1}^{*} \ldots, s_{m}^{*}\right\}$. Put $S_{j}=S^{*} s_{0}^{j}$. Let $d_{j}=$ $\min _{s \in S_{j}} d\left(s p_{0}, p_{0}\right)$. By (3.5) we have that $d_{j} \leq l$. Since subsets $S_{j}$ are disjoint the intersection $B\left(p_{0}, 2 l / \delta^{2}\right) \cap S_{j}$ is non-empty for every positive integer $j$. Therefore the semigroup $S$ does not acts properly discontinuously on $\mathbb{R}^{n}$. Contradiction.

Now we will prove the Margulis conjecture.

Proof. Let $G$ be the Zariski closure of the semigroup $S$. Let us show that $G \cap$ $\mathbb{R}^{n}$ is a finite index subgroup in $G$. It is enough to show that if the group $G$ is connected then $G \subseteq \mathbb{R}^{n}$. From Proposition 3.4 follows that the group $\overline{\ell(S)}$ is solvable. This group is compact and connected, therefore it is an abelian group. It is well known that a finitely generated linear group contains torsion free subgroup of a finite index. Therefore by above arguments we can and will assume that $S$ is torsion free. Thus for two different regular elements $s_{1}$ and $s_{2}$ from $S$ we have $V^{0}\left(s_{1}\right)=V^{0}\left(s_{2}\right)$. Then by lemma $3.1 V^{0}(s)=\mathbb{R}^{n}$ for every regular element of $S$. Consequently for every element $s \in S$ we have $\ell(s)=1$. Therefore the connected component $G^{0} \subseteq \mathbb{R}^{n}$.

Let $S_{0}=G^{0} \cap S$. It follows from Lemma 3.2 since $G^{0}$ is a subgroup of finite index in $G$ that $C\left(S^{0}\right)=\mathbb{R}^{n}$. On the other hand the semigroup $S^{0}$ contains only translations and acts properly discontinuously. Therefore $S^{0}$ is a group. It is clear that if a subsemigroup of finite index in semigroup is a group then the semigroup is a group which proves the statement. 


\section{REFERENCES}

[A ] H. Abels, Proximal Linear Maps, (in this volume)

[AMS 1] H. Abels, G.A. Margulis and G.A. Soifer, Semigroups containing proximal linear maps, Israel J. of Math. 91 (1995) 1-30

[AMS 2] -, On the Zariski closure of the linear part of a properly discontinuous group of affine transformations, J. Diff. Geom. 60, (2003),2, 314-335

[B] A. Borel, Linear Algebraic Groups, Springer Verlag.

[BG 1] E. Breuillard, T. Gelander, On dense free subgroups of Lie groups, J. Algebra 261 (2003), no. 2, 448-467.

[BG 2] E. Breuillard, T. Gelander, Topological Tits alternative, Ann of Math (to appear).

[GGl] T. Gelander, Y. Glasner, Infinite Primitive Groups, GAFA.

[DPSS] J.D. Dixon, L. Pyber, A. Seress, A. Shalev, Residual properties of free groups and probabilistic methods, J. Reine Angew. Math. 556 (2003), 159-172.

[MS 1] G.A. Margulis, G.A. Soifer, The criterion of the existence of maximal subgroups of infinite index in a finitely generated linear group, Soviet Math. Dokl. 18, N3 (1977), 847-851

[MS 2] G.A. Margulis, G.A. Soifer, Nonfree maximal subgroups of infinite index in the group $S L_{n}(\mathbb{Z})$, Uspekhi Math. Nauk, 34,4(208) (1979) 203-204

[MS 3] G.A. Margulis, G.A. Soifer, Maximal subgroups of infinite index in finitely generated linear group, J. Algebra 69, No 1., (1981), 1 -23

[P] L. Pyber, Groups enumeration and where it leads us, Prog. Math. Birkhauser, 169 (1996), 187-199

[R] M.S. Raghunathan, Discrete subgroups of a Lie groups, Springer -Verlag, 1972

[S] G.A. Soifer, The linear part of a discontinuously acting euclidean semigroup, J. Algebra, 250 (2002), 647-663.

[SV] G. Soifer, T. Venkataramana, Finitely generated pro-finitely dense free groups in higher rang semisimple groups, Transform. Groups 5 (2000), no. 1, 93-100

[T] J. Tits, Free subgroups in linear groups, J. Algebra 20 (1972)

[V] T.N. Venkataramana, J. of Algebra 108, (1987), 325-339

[W] B. Weisffeiler, Strong approximation for Zariski dense subgroups of semisimple algebraic groups, Ann. of Math. 120 (1984), 271-315.

\section{G.A. Soifer}

Dept. of Mathematics

Bar-Ilan University

52900 Ramat-Gan

Israel

E-mail: soifer@macs.biu.ac.il 